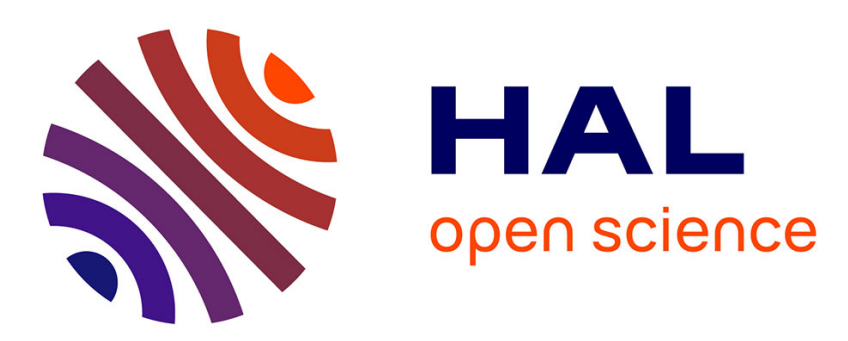

\title{
Action d'énergies lumineuses élevées sur la photosynthèse et la photorespiration chez le blé
} Ali Vahap Katkat, Françoise Ferron, Alain Coudret, Claude Costes

\section{To cite this version:}

Ali Vahap Katkat, Françoise Ferron, Alain Coudret, Claude Costes. Action d'énergies lumineuses élevées sur la photosynthèse et la photorespiration chez le blé. Agronomie, 1986, 6 (4), pp.337-344. hal-00884883

\section{HAL Id: hal-00884883 https://hal.science/hal-00884883}

Submitted on 1 Jan 1986

HAL is a multi-disciplinary open access archive for the deposit and dissemination of scientific research documents, whether they are published or not. The documents may come from teaching and research institutions in France or abroad, or from public or private research centers.
L'archive ouverte pluridisciplinaire HAL, est destinée au dépôt et à la diffusion de documents scientifiques de niveau recherche, publiés ou non, émanant des établissements d'enseignement et de recherche français ou étrangers, des laboratoires publics ou privés. 


\title{
Action d'énergies lumineuses élevées sur la pho- tosynthèse et la photorespiration chez le blé
}

\author{
Ali Vahap KATKAT, Françoise FERRON $\left({ }^{*}\right)$, Alain COUDRET $\left({ }^{* *}\right)$ \& Claude COSTES $\left({ }^{*}\right)$ \\ Uludag Üniversitesi, Ziraat Fakültesi, Bursa, Turquie. \\ (*) I.N.R.A., I.N.A.-P.G., Laboratoire de Chimie biologique et de Photophysiologie, F 78850 Thiverval- \\ Grignon. \\ (**) Faculté des Sciences, Laboratoire de Biologie et Physiologie végétales, F 72017 Le Mans Cedex.
}

Mots clés additionnels : Energie lumineuse, stomates, échanges gazeux et enzymes de la photosynthèse, métabolismes photosynthétique et photorespiratoire; Triticum aestivum.

Effect of high irradiance on photosynthesis and photorespiration in wheat.

Stomatal opening, net photosynthesis, enzyme activities of RuBPC and PEPC, and photosyntheticphotorespiratory metabolisms were studied in wheat under increasing irradiance from 40 to $2000 \mu$ moles. $\mathrm{m}^{-2} \cdot \mathrm{s}^{-1}$. The studies on the first three confirmed data well known in wheat. Results on relative variation in metabolic flux suggested that photorespiration plays a positive part in production, in protecting chloroplast structures and diversifying photosynthetic biosynthesis.

Additional key words : Light energy, stomata, gaseous exchange and enzymes of photosynthesis, photosynthetic and photorespiratory metabolism, Triticum aestivum.

\section{ABRÉVIATIONS :}

RuBPC : ribulose-1,5-bisphosphate-carboxylase ;

PEPC : phosphoénolpyruvate-carboxylase ;

RuBP : ribulose-1,5-bisphosphate ;

TCA : acide trichloracétique ;
PEP : phosphoénolpyruvate ;

EDTA : acide éthylènediaminetétraacétique ;

PGA : 3-phosphoglycérate ;

DHAP : dihydroxyacétone phosphate.

\section{INTRODUCTION}

Depuis BLACKMAN (1905), l'étude des variations de l'intensité de la photosynthèse (mesurée par la méthode des échanges gazeux) en fonction de l'éclairement met en évidence un effet de saturation lumineuse. Cet effet a fait l'objet de nombreux travaux repris plus tard sur diverses plantes vertes, notamment par BJÖRKMAN (1968), WILD (1979), GAUDILLĖRE (1979).

La saturation lumineuse apparaît pour des éclairements plus ou moins élevés selon l'espèce considérée et les autres facteurs climatiques de l'environnement : une plante de type $C_{3}$ est plus facilement saturée en lumière qu'une plante de type $\mathrm{C}_{4}$.

Si l'effet de l'énergie lumineuse incidente a été bien étudié au plan de la régulation stomatique des échanges gazeux et des activités enzymatiques de la photosynthèse (KUIPER, 1961; BOWES et al., 1972; BUCHANAN, 1980 par exemple), il n'en est pas de même pour le métabolisme carboné, notamment à plusieurs valeurs d'éclairements saturants (FERRON $e t$ al., 1982).

Le but de ce travail est donc de définir, en relation avec les échanges de $\mathrm{CO}_{2}$ photosynthétiques et le comportement stomatique, les réactions métaboliques 
d'un blé (espèce de type $C_{3}$ ) soumis à une gamme croissante d'énergies incidentes comprises entre 40 et 2000 umoles. $\mathrm{m}^{-2} . \mathrm{s}^{-1}$.

\section{MATÉRIEL ET MÉTHODES}

Le blé (Triticum aestivum L., cv. « Champlein ») est cultivé sur vermiculite arrosée de solution nutritive (CC' : COÏC \& LESAINT, 1973). L'alternance de température jour/nuit est de $20 / 15^{\circ} \mathrm{C}$ et l'humidité relative est de $80 \mathrm{p} .100$. La photopériode est de $16 \mathrm{~h}$ et les plantes reçoivent, au niveau du collet, $200 \mu$ moles $. \mathrm{m}^{-2} . \mathrm{s}^{-1}$ de lumière incidente (400$700 \mathrm{~nm}$, tubes fluorescents General Electric Cool White, mesures d'éclairement avec le Quantum Meter LICOR). Les expériences sont effectuées sur la $2^{\mathrm{e}}$ feuille adulte, au stade 4 feuilles, sous 40,200 , 400,1000 et $2000 \mu$ moles. $\mathrm{m}^{-2} \cdot \mathrm{s}^{-1}$ de lumière incidente (lampe XBF 6000 OSRAM).

\section{A. Mouvements stomatiques}

Des poromètres à hydrogène (LOUGUET, 1965 ; COUDRET \& LOUGUET, 1980) sont montés sur les feuilles à étudier ; ils sont balayés par l'air ambiant du laboratoire, à l'obscurité $\left(+\mathrm{H}_{2} \mathrm{O}\right.$ : humidité relative, 40 p. $\left.100 ;+\mathrm{CO}_{2}: 320 \mathrm{vpm}\right)$ pendant $8 \mathrm{~h}$, puis une énergie lumineuse de $40 \mu$ moles. $\mathrm{m}^{-2} \cdot \mathrm{s}^{-1}$ est appliquée sur les feuilles jusqu'à ce que ces dernières présentent une ouverture stomatique constante. L'énergie lumineuse est ensuite portée à $200 \mu$ moles. $\mathrm{m}^{-2} \cdot \mathrm{s}^{-1}$ (jusqu'à ouverture constante), puis à 400,1000 et $2000 \mu$ moles. $\mathrm{m}^{-2} \cdot \mathrm{s}^{-1}$. Lorsque l'ouverture stomatique est stabilisée sous cet éclairement, l'air ambiant des feuilles est remplacé par de l'air sec et dépourvu de $\mathrm{CO}_{2}\left(-\mathrm{H}_{2} \mathrm{O},-\mathrm{CO}_{2}\right)$ afin d'obtenir l'ouverture maximale potentielle des stomates. Ensuite, toujours en air sec dépourvu de $\mathrm{CO}_{2}$, les feuilles sont soumises à 1000 , puis 400,200 et $40 \mu$ moles. $\mathrm{m}^{-2} \cdot \mathrm{s}^{-1}$ d'énergie lumineuse incidente. Enfin, les plantes sont remises à l'obscurité, les feuilles étant de nouveau balayées par de l'air ambiant de la chambre de culture.

\section{B. Photosynthèse nette}

Après $15 \mathrm{j}$ de culture, des pieds de blé au stade 4 feuilles sont placés dans une enceinte fermée de $15 \mathrm{~cm}^{3}$ en plexiglass, dans laquelle un ventilateur brasse l'air. L'enceinte est balayée à raison de $601 . \mathrm{h}^{-1}$ par de l'air dont la teneur en $\mathrm{CO}_{2}$ est de $320 \mathrm{vpm}$. A la sortie, un analyseur de gaz par infrarouge permet de doser en permanence le $\mathrm{CO}_{2}$ présent dans l'air. Toutes les heures, pendant $2 \mathrm{mn}$, une prise d'air est effectuée sur le circuit d'arrivée d'air dans l'enceinte et la teneur en $\mathrm{CO}_{2}$ est contrôlée (COUDRET et al., 1980).

$\mathrm{Ce}$ dispositif permet de mesurer la quantité de $\mathrm{CO}_{2}$ absorbée par la plante entière, lorsqu'on la soumet à divers éclairements $(40,200,400,1000$, $2000 \mu$ moles. $\left.\mathrm{m}^{-2} . \mathrm{s}^{-1}\right)$. Les résultats sont rapportés à la surface foliaire mise en expérience et mesurée par la méthode héliographique.

\section{Activités enzymatiques de la RuBPC et de la PEPC}

La méthode de CHEN et al. (1971), modifiée par PASSERA \& Albuzio (1978), a été utilisée sur le surnageant d'un broyat de feuilles effectué en présence de tampon Tris $\mathrm{HCl}(0,1 \mathrm{M})$ à $4{ }^{\circ} \mathrm{C}$ et à $\mathrm{pH} 8,0$, contenant $10 \mathrm{mM}$ de dithiothreitol, puis passé sur Séphadex G 25 .

\section{Mesure de l'activité RuBPC}

Le milieu réactionnel contient $10 \mu$ moles de tampon Tris, $\mathrm{pH} 8,3,1 \mu$ mole de $\mathrm{MgCl}_{2}, 1 \mu$ mole de dithiothréitol, $0,17 \mu$ mole de RuBP, $10 \mu$ moles de $\mathrm{NaHCO}_{3}$, $0,011 \mu$ mole de $\mathrm{NaH}{ }^{14} \mathrm{CO}_{3}(74 \mathrm{kBq})$ et $40 \mu \mathrm{l}$ d'extrait foliaire dans un volume total de $0,1 \mathrm{ml}$. Le milieu réactionnel est incubé pendant $5 \mathrm{mn}$ à $30^{\circ} \mathrm{C}$. La réaction est stoppée par $0,1 \mathrm{ml}$ de TCA à $10 \mathrm{p} .100$ $(\mathrm{P} / \mathrm{V})$ qui permet de convertir $\mathrm{H}^{14} \mathrm{CO}_{3}^{-}$non incorporé en $\mathrm{CO}_{2}$ et un courant d'azote dans le milieu pendant $1 \mathrm{mn}$ élimine le $\mathrm{CO}_{2}$ en excès. $0,01 \mathrm{ml}$ est prélevé du milieu et sa radioactivité est mesurée par scintillation liquide.

\section{Mesure de l'activité PEPC}

Le milieu réactionnel contient 5,0 $\mu$ moles de tampon Tris $\mathrm{HCl}, \mathrm{pH} 8,3,0,5 \mu$ mole de $\mathrm{MgCl}_{2}, 0,5 \mu$ mole de dithiothréitol, $0,5 \mu$ mole de glutamate de sodium, $1 \mu$ mole de phosphoénolpyruvate, $1,5 \mu$ moles d'EDTA (forme acide), $0,5 \mu$ mole de $\mathrm{NaHCO}_{3}, 0,011 \mu$ mole de $\mathrm{NaH}{ }^{14} \mathrm{CO}_{3}(74 \mathrm{kBq})$. La réaction commence par l'addition de $60 \mu \mathrm{l}$ d'extrait foliaire dans un volume final de $0,1 \mathrm{ml}$. L'incubation est de $10 \mathrm{mn}$ à $30^{\circ} \mathrm{C}$. L'arrêt de la réaction et le comptage de la radioactivité sont identiques à ceux décrits dans le cas de la RuBPC.

Les résultats sont rapportés à la masse de matière fraîche, aux chlorophylles (MAC KINNEY, 1941) et aux protéines foliaires (LOWRY et al., 1951 ; HARTREE, 1972 ; GALZIN, 1979).

\section{Métabolisme photosynthétique}

Avant de séparer les feuilles de leurs pieds de blé, les plantes sont conditionnées pendant $1 \mathrm{~h}$ sous les éclairements étudiés: $40,200,400,1000$ ou $2000 \mu$ moles . $\mathrm{m}^{-2} . \mathrm{s}^{-1}$.

Les feuilles sont ensuite coupées et conditionnées pendant $10 \mathrm{mn}$ sous le même éclairement à $20^{\circ} \mathrm{C}$, dans une petite chambre à photosynthèse $\left(15 \mathrm{~cm}^{3}\right)$. Ces feuilles sont alors balayées avec de l'air identique à celui du préconditionnement, mais enrichi en ${ }^{14} \mathrm{CO}_{2}$ (radioactivité spécifique : $370 \mathrm{MBq} \mathrm{s}^{-1} \mathrm{mmol}^{-1}$ ) pendant une durée variable de charge de $10 \mathrm{~s}, 30 \mathrm{~s}, 60 \mathrm{~s}$, $120 \mathrm{~s}, 240 \mathrm{~s}$ et $360 \mathrm{~s}$ (COUdRET et al., 1981). Des expériences de chasses sont également réalisées : après $10 \mathrm{~s}$ de fourniture d'air enrichi en ${ }^{14} \mathrm{CO}_{2}$, les feuilles sont balayées par l'air ambiant, dépourvu de ${ }^{14} \mathrm{CO}_{2}$, pendant $30,60,120,200,400$ et $960 \mathrm{~s}$.

Les feuilles sont alors plongées dans l'azote liquide avant extraction des oses phosphorylés, des acides organiques et des acides aminés par de l'éthanol aqueux à 50 p. $100(\mathrm{v} / \mathrm{v})$, en présence de DOWEX 50 (forme $\mathrm{H}^{+}$), de $\mathrm{NaF}\left(10^{-4} \mathrm{M}\right)$ et de $\mathrm{KBH}_{4}$. Les produits primaires de carboxylation sont séparés sur cou- 
che mince (cellulose MN 300) à partir de fractions aliquotes d'extrait brut concentré (correspondant à environ $5 \mathrm{mg}$ de matière fraîche) en combinant deux méthodes, électrophorèse à haut voltage et séparation chromatographique : méthode de SCHÜRMANN (1969), modifiée par FERRON et al. (1978).

Les mesures de radioactivité ${ }^{14} \mathrm{C}$ incorporée sont effectuées par scintillation liquide, d'une part, sur la totalité de la matière organique soluble à partir d'une fraction aliquote de l'extrait brut, d'autre part, sur les métabolites solubles isolés après identification autoradioagraphique, enfin sur le résidu insoluble, après combustion par l'oxygène (Oxymat). En ce qui concerne le résidu insoluble, les résultats ne sont pas pris en compte dans la discussion, car la moyenne des mesures $\overline{\mathrm{m}}$, en pour cent de la radioactivité incorporée dans la matière organique totale brûlée dans les mêmes conditions, est égale à $3,2 \pm 1,2$ pour un intervalle de sécurité de 95 p. 100 .

Les résultats sont exprimés de la façon suivante pour le temps de charge en ${ }^{14} \mathrm{CO}_{2}$ et le temps de chasse éventuel en ${ }^{12} \mathrm{CO}_{2}$ considéré :

- la radioactivité incorporée dans la totalité de la matière organique soluble est exprimée en coups $/ \mathrm{mn}$ dans $1 \mathrm{mg}$ de matière fraîche ;

- la radioactivité incorporée dans les métabolites solubles est exprimée soit en coups/mn dans $1 \mathrm{mg}$ de matière fraîche, soit en pour cent de la radioactivité incorporée dans la totalité de la matière organique soluble.

On a considéré le PGA, le DHAP, les oses mono et bisphosphates comme métabolites représentatifs du cycle de Calvin, la glycine, la sérine et le glycérate comme marqueurs du carbone dans le métabolisme photorespiratoire, le malate et l'aspartate comme représentatifs des $\beta$-carboxylations observées à la lumière.

L'interprétation des données expérimentales d'ordre cinétique concernant le marquage des premiers composés de la photosynthèse par le ${ }^{14} \mathrm{C}$ est effectuée en utilisant le modèle bicompartimental proposé par GALMICHE (1973) : travaillant en régime photosynthétique stationnaire, le flux de carbone entrant est égal au flux sortant pour chaque compartiment et est égal à la vitesse de consommation de carbone sous forme de $\mathrm{CO}_{2}$ par les feuilles. Dans ces conditions, pour les temps courts de marquage, les compartiments ne sont pas saturés en ${ }^{14} \mathrm{C}$; la radioactivité mesurée dans chaque groupe de métabolites permet d'estimer la part du flux de carbone qui emprunte la voie métabolique considérée.

L'ensemble des manipulations a été répété 6 fois.

\section{RÉSULTATS}

\section{A. Ouverture stomatique et photosynthèse nette}

Plus l'énergie lumineuse incidente est élevée, plus l'ouverture stomatique est grande (fig. 1). De plus, l'absence de $\mathrm{CO}_{2}$ et de vapeur d'eau dans l'air accentue cette ouverture d'environ 25 p. 100. En air normal, les basses énergies sont relativement plus efficaces que les fortes. Ainsi, sous $40 \mu$ moles. $\mathrm{m}^{-2} \cdot \mathrm{s}^{-1}$,

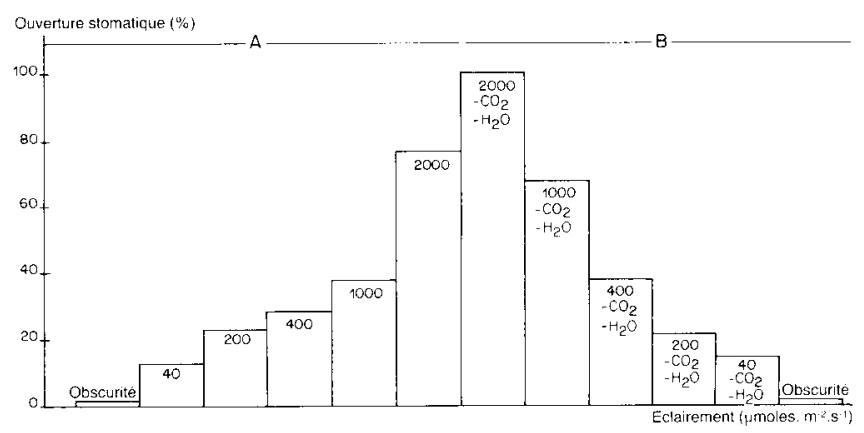

Figure 1

Courbe représentative des variations du degré d'ouverture stomatique (en pour cent de l'ouverture maximale potentielle) mesuré sur la $2^{e}$ feuille adulte d'un blé de $15 j$ :

A) en fonction de l'énergie lumineuse incidente en air normal (humidité relative : $40 \mathrm{p} .100$; concentration en $\mathrm{CO}_{2}: 320 \mathrm{vpm}$ ); $B)$ en fonction de l'énergie lumineuse incidente en air sec et sans $\mathrm{CO}_{2}$.

Variation in the degree of stomatal opening (per cent of maximal potential aperture) measured on the second adult leaf from 15-day old wheat:

A) in relation to incident irradiance in normal air (relative humidity : $40 \% ; \mathrm{CO}_{2}$ concentration : $320 \mathrm{vpm}$ );

B) in relation to incident irradiance in dry air without $\mathrm{CO}_{2}$.

l'ouverture stomatique représente 17 p. 100 de ce qu'elle est sous $2000 \mu$ moles. $\mathrm{m}^{-2} \cdot \mathrm{s}^{-1}$; pour un éclairement 50 fois supérieur, l'ouverture n'est donc multipliée que par 5,9. Par contre, entre 1000 et $2000 \mu$ moles. $\mathrm{m}^{-2} . \mathrm{s}^{-1}$, l'ouverture stomatique augmente proportionnellement avec l'énergie lumineuse incidente.

Le taux de photosynthèse nette n'augmente plus proportionnellement avec le niveau d'éclairement entre 200 et $1000 \mu$ moles. $\mathrm{m}^{-2} \cdot \mathrm{s}^{-1}$; puis, à partir de cette dernière valeur, l'absorption nette de $\mathrm{CO}_{2}$ tend vers un palier qui indique une saturation lumineuse des échanges gazeux (fig. 2).

Il n'y a pas corrélation directe entre photosynthèse apparente et ouverture stomatique.

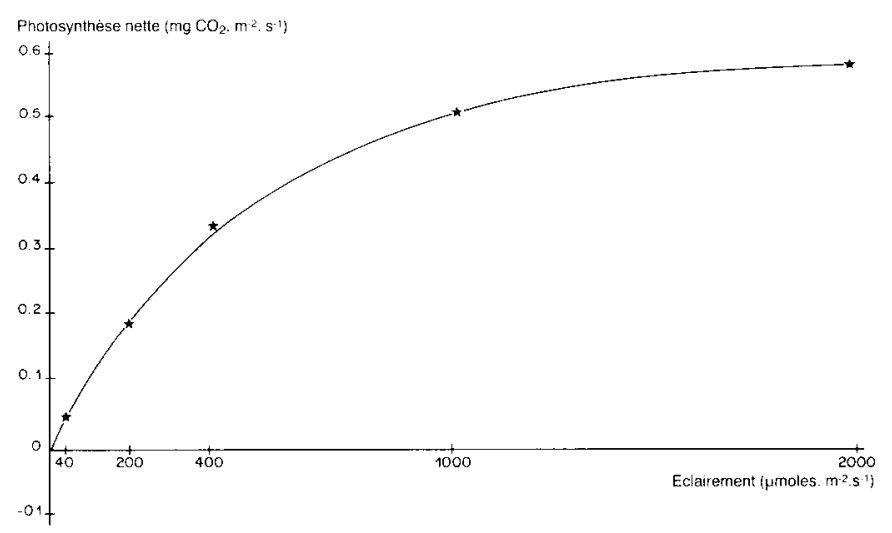

Figure 2

Photosynthèse nette de 12 plantes entières mesurée en fonction de l'énergie lumineuse incidente sur un pot de culture de blé âgé de $15 \mathrm{j}$.

Net photosynthesis of 12 whole plants measured in relation to incident irradiance from 15-day old wheat. 


\section{B. Activités enzymatiques}

Quel que soit le mode d'expression des résultats, l'activité enzymatique de la RuBPC, mesurée in vitro, n'est très fortement intensifiée par la lumière que pour $2000 \mu$ moles. $\mathrm{m}^{-2} \cdot \mathrm{s}^{-1}$, par rapport aux autres énergies incidentes étudiées. En ce qui concerne la PEPC, l'activité enzymatique n'apparaît par corrélée significativement avec le niveau d'éclairement (fig. 3).

Il convient de souligner le fait que ces mesures d'activité enzymatique in vitro ne permettent pas d'établir de comparaisons entre l'activité in vivo de la RuBPC et celle de la PEPC. Les conditions expérimentales déterminent trop étroitement les valeurs mesurées sur les extraits cellulaires. Tout au plus peuton comparer les activités mesurées pour une même enzyme provenant de feuilles ayant subi différents traitements physiologiques.

\section{Incorporation du ${ }^{14} \mathrm{C}$ dans la matière organique soluble}

A partir des données de la figure 4 et du tableau 1, il ressort que, pour toutes les énergies lumineuses incidentes, lorsque le temps de charge avec ${ }^{14} \mathrm{CO}_{2}$ augmente, la radioactivité incorporée varie linéairement avec lui, le coefficient de corrélation étant significatif $(\mathrm{p}<0,001)$.
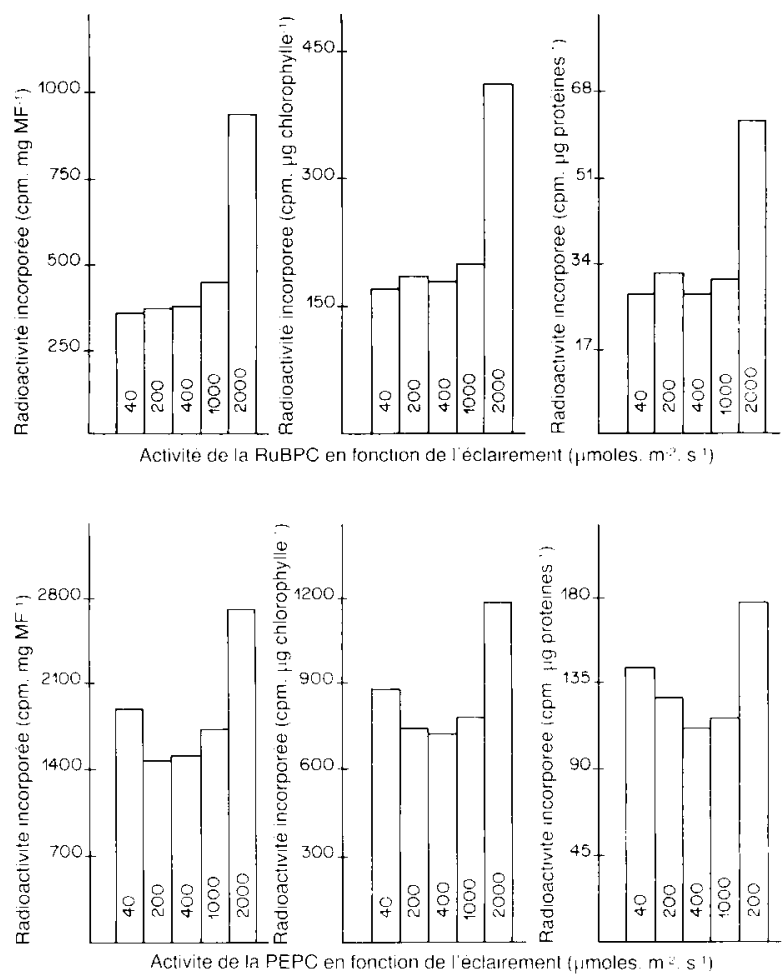

Figure 3

Activité de la RuBPC et de la PEPC en fonction de l'énergie lumineuse incidente: les résultats provenant d'expériences in vitro sont rapportés à la masse de matière fraîche, à la teneur en chlorophylles et en protéines foliaires.

Activity of RuBPC and of PEPC in relation to incident irradiance. results from in vitro experiments expressed on the basis of fresh matter, chlorophyll and leaf protein content.

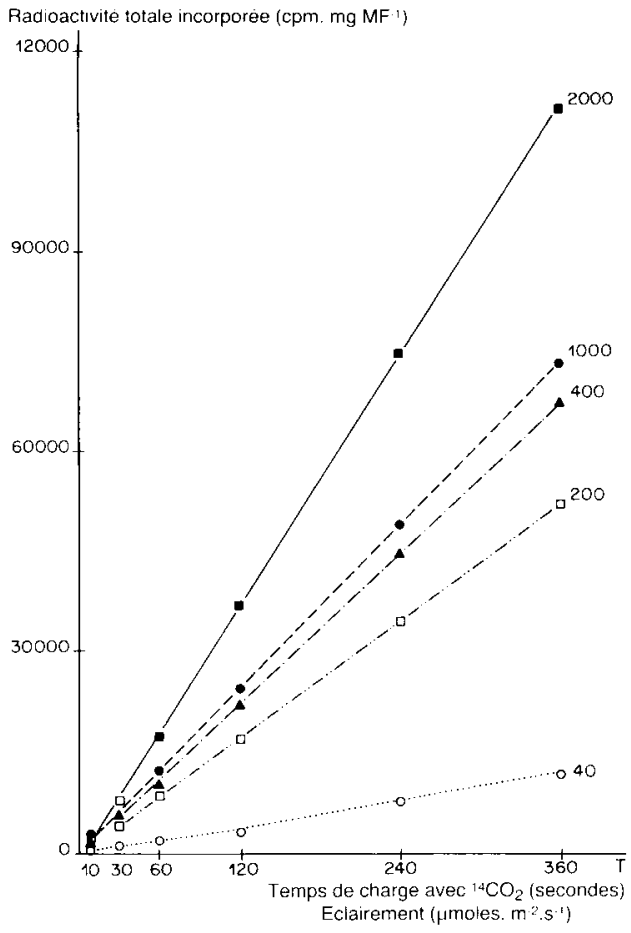

Figure 4

Radioactivité incorporée dans la matière organique soluble, en fonction du temps de charge avec ${ }^{14} \mathrm{CO}_{2}$ et de l'énergie lumineuse incidente.

Radioactivity incorporated into soluble organic matter in relation to ${ }^{14} \mathrm{CO}_{2}$ pulse time and incident light energy.

\section{TABLEAU}

Equations des droites de régression et coefficients de corrélation linéaire pour la radioactivité totale incorporée dans la matière organique soluble en fonction de l'énergie lumineuse incidente (cf. fig. 4).

Regression equations and linear correlation coefficients for the total radioactivity incorporated into soluble organic matter, in relation to incident light energy (see fig. 4).

Energie lumineuse Droites de régression pour la incidente radioactivité totale incorporée Coefficient de $\left(\mu\right.$ moles $\left.\cdot \mathrm{m}^{-2} \cdot \mathrm{s}^{-1}\right) \quad$ en fonction du temps $\mathrm{x}$ corrélation $(\mathrm{r})$

$\begin{array}{rrr}40 & 82,2+36,6 \times & r=0,98 \\ 200 & 173,0+151,3 \times & r=0,99 \\ 400 & -167,9+193,2 \times & r=0,96 \\ 1000 & -205,3+211,0 \times & r=0,98 \\ 2000 & -968,0+323,4 \times & r=0,99\end{array}$

\section{Incorporation du ${ }^{14} \mathrm{C}$ dans les différents assimilats de la photosynthèse}

Il faut noter que les mesures du métabolisme photosynthétique sont effectuées dans des conditions de température et d'humidité ambiantes maintenues constantes et non limitantes ; seul le niveau d'éclairement varie et la régulation de l'ouverture stomatique est de nature "photoactive " et non "hydroactive " pendant la durée des manipulations pour la mesure (1 h 30 environ). En conséquence, on peut avancer que les variations mesurées dans les flux de ${ }^{14} \mathrm{C}$ assimilés correspondent à des variations dans la production de matière sèche avec le niveau d'éclairement. 


\section{Métabolites du cycle de Calvin}

De la figure $5 a$, il ressort qu'il y a beaucoup plus de radioactivité incorporée dans les métabolites du cycle de Calvin sous 2000 que sous $40 \mu$ moles. $\mathrm{m}^{-2}$. $\mathrm{s}^{-1}$ d'énergie lumineuse incidente. Mais pour 200, 400 et $1000 \mu$ moles. $\mathrm{m}^{-2} . \mathrm{s}^{-1}$, la radioactivité incorporée est sensiblement la même et se trouve comprise entre les valeurs extrêmes observées pour 40 et $2000 \mu$ moles. $\mathrm{m}^{-2} . \mathrm{s}^{-1}$.

Notamment pour des temps de charge relativement longs (à partir de $120 \mathrm{~s}$ ), la part relative du ${ }^{14} \mathrm{C}$ incorporée dans ces métabolites apparaît plus importante sous $40 \mu$ moles. $\mathrm{m}^{-2} . \mathrm{s}^{-1}$ de lumière incidente que sous les éclairements les plus élevés (fig. 6). Les cinétiques de chasse du ${ }^{14} \mathrm{C}$ par le ${ }^{12} \mathrm{CO}_{2}$ montrent également que la radioactivité réside proportionnellement plus longtemps dans ces métabolites sous l'éclairement le plus faible (fig. 7).

\section{Métabolites photorespiratoires}

Plus l'énergie lumineuse incidente est élevée, plus il s'incorpore de ${ }^{14} \mathrm{C}$ dans l'ensemble des métabolites marqueurs de la voie de TOLBERT (1971), tant en valeur absolue qu'en valeur relative (fig. $5 b$, fig. 6). Cependant, pour $2000 \mu$ moles. $\mathrm{m}^{-2} \cdot \mathrm{s}^{-1}$ d'énergie lumineuse incidente, on note une accumulation de ${ }^{14} \mathrm{C}$ dans la glycine par rapport à ce qu'on observe dans la sérine (fig. $5 c$ et $d$ ).
$\mathrm{Au}$ cours des expériences de chasse du ${ }^{14} \mathrm{C}$ par le ${ }^{12} \mathrm{CO}_{2}$, l'évolution de la radioactivité dans les métabolites photorespiratoires se stabilise à partir de $120 \mathrm{~s}$ pour les plus faibles éclairements $(40 \mathrm{et}$ $200 \mu$ moles. $\left.\mathrm{m}^{-2} . \mathrm{s}^{-1}\right)$; puis la décharge en ${ }^{14} \mathrm{C}$ de ce groupe de métabolites se fait parallèlement à celle mesurée pour les métabolites du cycle de Calvin sous 400 et $1000 \mu$ moles. $\mathrm{m}^{-2} \cdot \mathrm{s}^{-1}$ avant de se stabiliser à nouveau après $200 \mathrm{~s}$, sous $2000 \mu$ moles. $\mathrm{m}^{-2} . \mathrm{s}^{-1}$ (fig. 7).

\section{Saccharose}

D'après la figure $5 e$, le taux de ${ }^{14} \mathrm{C}$ incorporé dans le saccharose ne varie pas proportionnellement avec le niveau d'énergie lumineuse incidente : ainsi, pour 1000 et $2000 \mu$ moles. $\mathrm{m}^{-2} \cdot \mathrm{s}^{-1}$, la radioactivité incorporée dans ce glucide est la même malgré le doublement de l'éclairement. Les figures 6 et 7 montrent en effet que la part relative $\mathrm{du}{ }^{14} \mathrm{C}$ incorporé dans le saccharose augmente peu à partir de $200 \mu$ moles. $\mathrm{m}^{-2} . \mathrm{s}^{-1}$, puis diminue entre 1000 et $2000 \mu$ moles. $\mathrm{m}^{-2} . \mathrm{s}^{-1}$. Les expériences de chasse du ${ }^{14} \mathrm{C}$ par le ${ }^{12} \mathrm{CO}_{2}$ montrent bien l'évolution opposée des cinétiques d'incorporation $\mathrm{du}{ }^{14} \mathrm{C}$ compatible avec une synthèse de saccharose à partir des métabolites du cycle de Calvin et, probablement, à partir des métabolites de la voie de Tolbert sous 400 et $1000 \mu$ moles. $\mathrm{m}^{-2} . \mathrm{s}^{-1}$ d'éclairement.
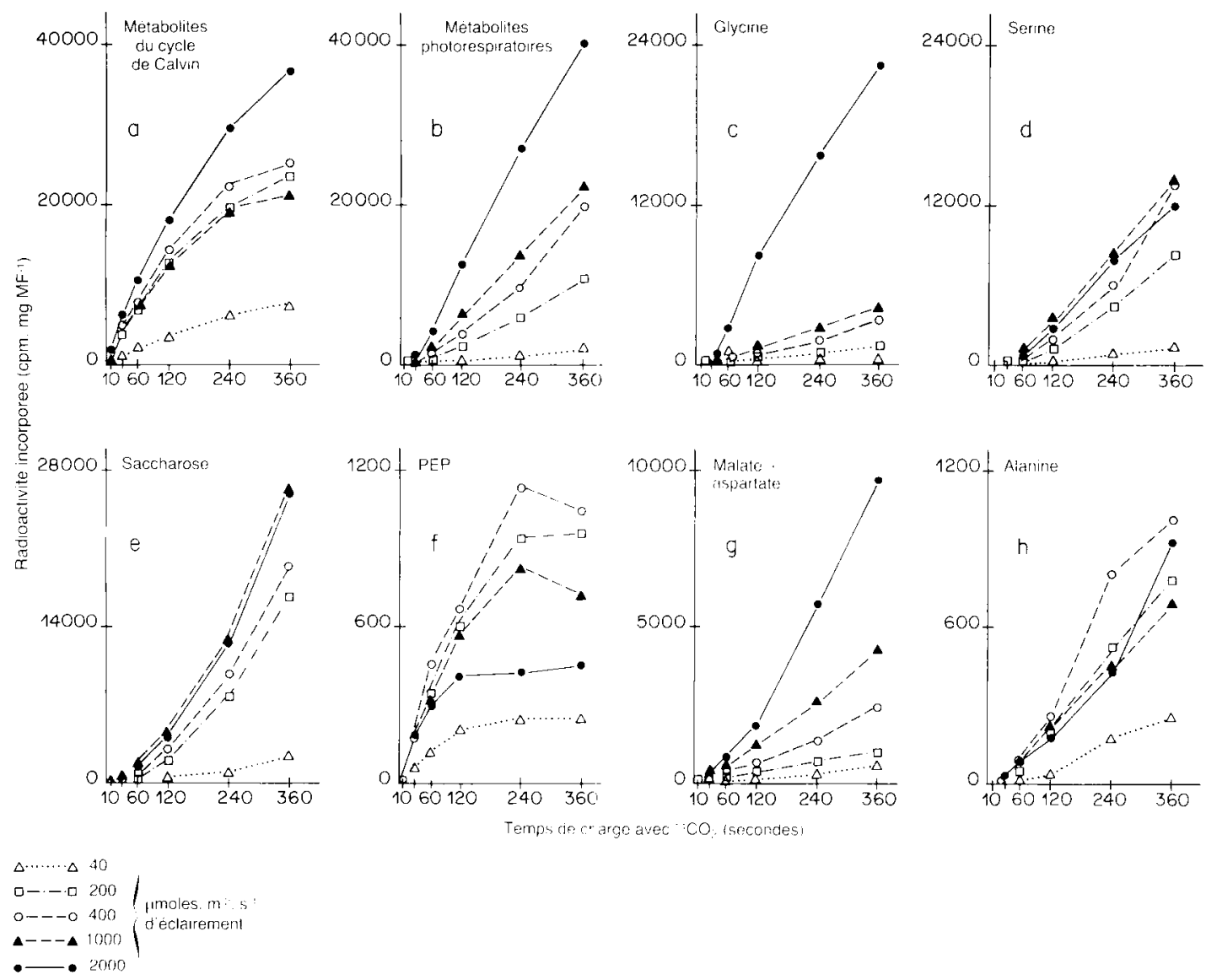

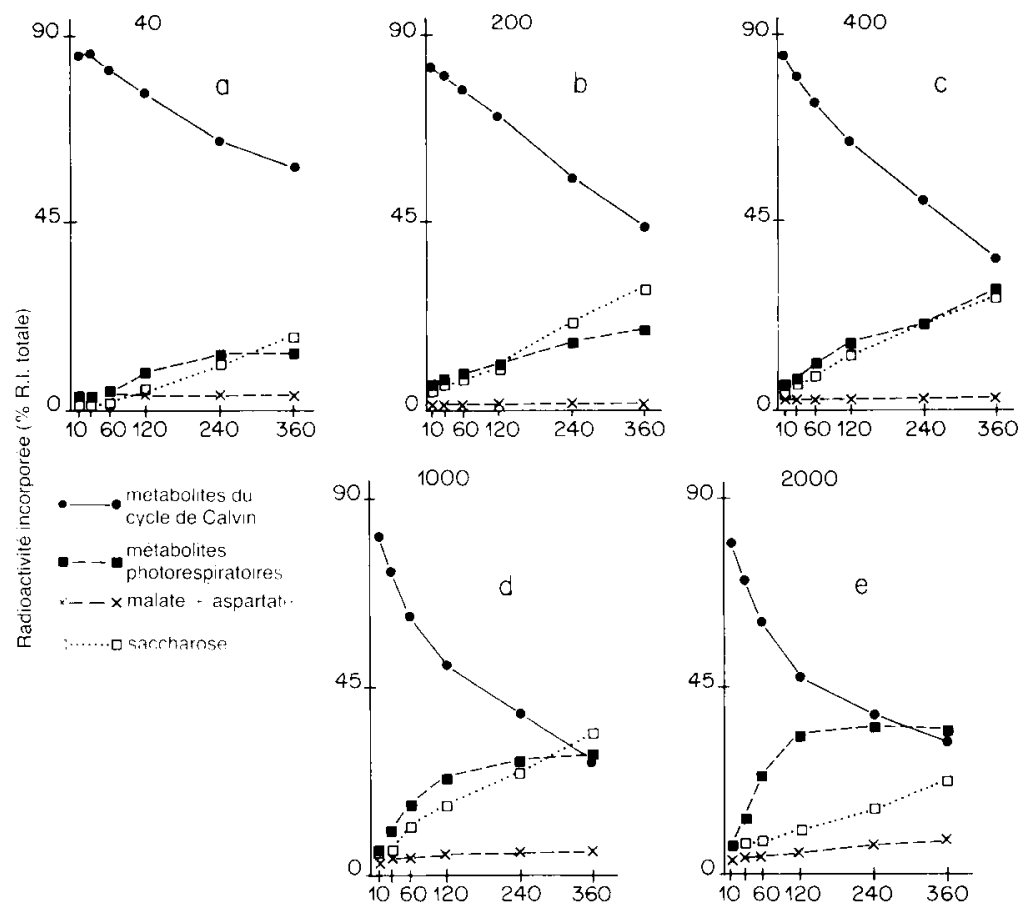

Temps de charge avec ${ }^{4} \mathrm{CO}$; (S) sous $40,200,400,1000,2000 \mu$ moles. $\mathrm{m}^{2} . \mathrm{s}^{1} \mathrm{~d}$ éclairement

Figure 6

Radioactivité incorporée (en p. 100 de la radioactivité totale incorporée dans la matière organique soluble) dans les différents assimilats photosynthétiques en fonction du temps de charge avec ${ }^{14} \mathrm{CO}_{2}$ et de l'énergie lumineuse incidente.
Radioactivity incorporated into various photosynthates in relation to ${ }^{14} \mathrm{CO}_{2}$ pulse time and incident light intensity.

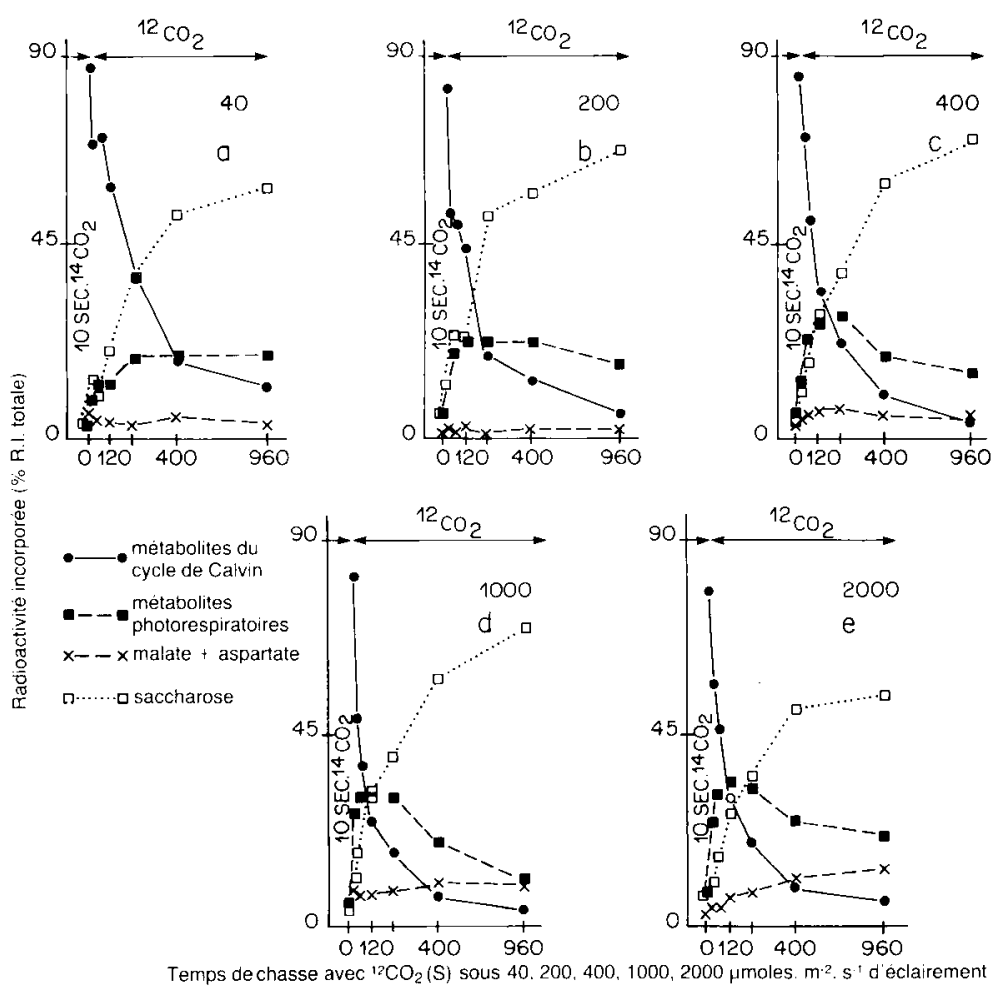

Figure 7

Après $10 \mathrm{~s}$ de temps de charge avec ${ }^{14} \mathrm{CO}_{2}$, radioactivité retrouvée dans les assimilats photosynthétiques en fonction du temps de chasse avec de l'air dépourvu de ${ }^{14} \mathrm{CO}_{2}$ et de l'énergie lumineuse incidente.
After $10 \mathrm{~s}$ of pulse time with ${ }^{14} \mathrm{CO}_{2}$, radioactivity measured in photosynthates in relation to chase time with ${ }^{14} \mathrm{CO}_{2}$-free air and to incident light energy. 


\section{PEP, malate et aspartate}

La radioactivité incorporée dans le PEP augmente avec la lumière jusqu'à $400 \mu$ moles. $\mathrm{m}^{-2} \cdot \mathrm{s}^{-1}$ d'énergie lumineuse incidente ; au-delà, elle diminue, d'autant plus que la lumière est plus forte (fig. $5 f$ ).

Que les mesures soient effectuées en valeurs absolues (fig. $5 g$ ) ou en valeurs relatives (fig. 6), plus l'énergie lumineuse incidente est grande plus il y a de ${ }^{14} \mathrm{C}$ incorporé dans (malate + aspartate).

\section{Alanine}

La radioactivité incorporée augmente avec la lumière jusqu'à $400 \mu$ moles. $\mathrm{m}^{-2} \cdot \mathrm{s}^{-1}$ d'énergie lumineuse incidente. Au-delà, le marquage est plus faible (fig. $5 h$ ).

\section{DISCUSSION ET CONCLUSION}

L'accent est porté sur les effets des énergies lumineuses élevées supérieures à $400 \mu$ moles. $\mathrm{m}^{-2} \cdot \mathrm{s}^{-1}$.

\section{A. Eclairement, échanges gazeux et incorporation du ${ }^{14} \mathrm{C}$ dans la matière organique soluble}

En ce qui concerne les échanges gazeux, il ressort que, du moins pour des énergies lumineuses élevées (de 400 à $2000 \mu$ moles. $\mathrm{m}^{-2} \cdot \mathrm{s}^{-1}$ ), l'ouverture stomatique est proportionnelle à l'énergie lumineuse incidente (fig. 1) : cette observation est comparable aux résultats de KUIPER (1961) sur tomate, haricot, jusquiame, et à ceux de FERRON et al. (1982) sur christophine. Les degrés d'ouverture stomatique, la photosynthèse nette et l'incorporation totale de ${ }^{14} \mathrm{C}$ dans les assimilats photosynthétiques mesurés en fonction de l'éclairement varient dans le même sens bien que non proportionnellement (fig. 1, 2 et 4) : l'absorption et l'assimilation du ${ }^{14} \mathrm{CO}_{2}$ sont corrélées avec l'ouverture stomatique pour la dépendance de l'intensité de l'éclairement. La non-proportionnalité entre les radioactivités incorporées dans les métabolites solubles foliaires et les niveaux d'éclairement utilisés peut provenir du fait que les pools de la plupart des composés analysés sont inégalement loin de la saturation en ${ }^{14} \mathrm{C}$ pour les temps de charge utilisés.

\section{B. Eclairement et incorporation de ${ }^{14} \mathrm{C}$ dans les diffé- rents assimilats de la photosynthèse}

\section{Métabolites du cycle de Calvin, de la voie de Tol- bert et saccharose}

D'après les figures 5,6 et 7, il apparaît une importante action de la lumière sur la distribution $\mathrm{du}^{14} \mathrm{C}$ fixé dans les métabolites de la photosynthèse et de la photorespiration.

Entre 200 et $1000 \mu$ moles. $\mathrm{m}^{-2} . \mathrm{s}^{-1}$ d'énergie lumineuse incidente, la quantité de ${ }^{14} \mathrm{C}$ incorporée dans les métabolites du cycle de Calvin semble être saturée pour la teneur normale de l'air en $\mathrm{CO}_{2}$. Or, dans le même temps, l'incorporation de ${ }^{14} \mathrm{C}$ dans le saccharose, principal produit de sortie du cycle de Calvin, augmente, de même que dans les métabolites photo- respiratoires : il est avancé l'hypothèse que ces derniers prendraient le relais des métabolites photosynthétiques pour la synthèse de saccharose. D'après les cinétiques de décharge de radioactivité des métabolites photosynthétiques et photorespiratoires au-delà de $120 \mathrm{~s}$ de chasse $\mathrm{du}{ }^{14} \mathrm{C}$ par le ${ }^{12} \mathrm{CO}_{2}$, les substrats du cycle de Calvin et ceux de la voie de Tolbert semblent être utilisés avec des vitesses comparables à partir de $400 \mu$ moles. $\mathrm{m}^{-2} . \mathrm{s}^{-1}$ d'éclairement (fig. $7 c, d$ et $e)$ : ces faits apportent un argument dans le sens d'un rôle anabolique qui peut être prêté à la voie du glycolate dans la mesure où on la réinsère dans le métabolisme des glucides et des acides aminés (CHAMPIGNY, 1980).

A partir de $1000 \mu$ moles. $\mathrm{m}^{-2} \cdot \mathrm{s}^{-1}$ de lumière incidente, la synthèse du saccharose n'augmente plus, alors que l'on constate une activation de la RuBPC et de la synthèse des métabolites intermédiaires du cycle de Calvin ainsi que des métabolites photorespiratoires (fig. 3 et $5 a$ et $b$ ); sous $2000 \mu$ moles. $\mathrm{m}^{-2} \cdot \mathrm{s}^{-1}$, la radioactivité $\mathrm{du}{ }^{14} \mathrm{C}$ s'accumule plus dans la glycine que dans la sérine. Au-delà de $1000 \mu$ moles. $\mathrm{m}^{-2} \cdot \mathrm{s}^{-1}$, le supplément d'énergie lumineuse ne se traduit donc plus par une synthèse accrue de saccharose, par suite d'une saturation du cycle de Calvin sous un éclairement élevé, mais on constate une orientation du métabolisme vers la production d'un acide aminé, la glycine (fig. $5 c, d$ et $e$ ).

Ces observations sont à rapprocher des faits suivants :

- Le taux d'incorporation du ${ }^{14} \mathrm{C}$ dans les métabolites photorespiratoires augmente régulièrement avec l'énergie lumineuse incidente (fig. $5 b$ ), ce qui est cohérent avec l'intensification des rejets de $\mathrm{CO}_{2}$ photorespiratoires décrits par CAVALIÉ (1981) lorsque l'éclairement croît.

- D'après les mesures de la photosynthèse nette (fig. 2), le flux maximal de $\mathrm{CO}_{2}$ fixé est atteint dès $1000 \mu$ moles. $\mathrm{m}^{-2} . \mathrm{s}^{-1}$ d'énergie lumineuse incidente.

Le peu d'effet de l'apport énergétique par des éclairements élevés sur la photosynthèse apparente semble provenir d'une dissipation de l'excès du pouvoir réducteur liée à l'augmentation relative du fonctionnement de la photorespiration, en conditions normales d'alimentation en $\mathrm{CO}_{2}$ et en $\mathrm{O}_{2}$, ce qui va dans le sens de l'hypothèse d'un rôle protecteur prêté à la photorespiration contre la photoinhibition (HEBER \& KRAUSE, 1980 ; OSMOND, 1981 ; Boyle \& KeYS, 1982 ; POWLES et al., 1984).

\section{Autres métabolites}

La synthèse de (malate + aspartate) augmente régulièrement avec la lumière incidente mais ne dépasse pas 10 p. 100 de la radioactivité totale incorporée dans le blé étudié, montrant bien que cette activité de synthèse ne correspond pas au métabolisme photosynthétique en $\mathrm{C}_{4}$. Les variations du taux de ${ }^{14} \mathrm{C}$ incorporé dans ces molécules apparaissent donc d'un intérêt mineur face à celles observées dans le cycle de Calvin et dans la voie photorespiratoire. Néanmoins un parallèle peut être établi entre la diminution du taux de ${ }^{14} \mathrm{C}$ mesuré dans l'alanine (via le PEP) à partir de $400 \mu$ moles. $\mathrm{m}^{-2}$. $\mathrm{s}^{-1}$ et l'augmentation du taux de ${ }^{14} \mathrm{C}$ mesuré dans les molécules en $\mathrm{C}_{4}$ (via le PEP) à partir du même niveau d'éclairement (fig. $5 g$ et $h$ ). 
Ces modulations des diverses composantes du métabolisme sous l'effet d'une haute énergie lumineuse incidente sont à comparer à celles déjà observées pour diverses conditions extrêmes de vie chez différentes plantes en $\mathrm{C}_{3}$ (COUdRET \& FERRON, 1982).

En conclusion, cette étude a permis de préciser, chez le blé, les contributions des différents métabolismes carbonés à l'élaboration de la matière organique à partir du $\mathrm{CO}_{2}$; d'autre part, ce travail constitue un apport mettant en évidence les flexibilités métaboliques qui peuvent conduire à l'adaptation d'un végétal photosynthétique connu aux faibles et forts éclairements.

Reçu le 9 mai 1985. Accepté le 25 novembre 1985.

\section{REMERCIEMENTS}

Ce travail a pu être réalisé grâce au soutien du C.I.E.S. qui a couvert, en partie, le séjour de A. KATKAT dans le Laboratoire de Chimie biologique et de Photophysiologie de l'I.N.R.A. à Grignon.

\section{RÉFÉRENCES BIBLIOGRAPHIQUES}

Björkman O., 1968. Further studies on differentiation of photosynthetic properties in sun and shade ecotypes of Solidago virgaurea. Physiol. Plant., 21, 81-99.

Blackman F. F., 1905. Optima and limiting factors. Ann. Bot., 19, 281-295.

Boyle F. A., Keys A. J., 1982. Regulation of RuBP carboxylase activity associated with photoinhibition of wheat. Photosynth. Res., $3,105-111$.

Bowes G., Ogren W. L., Hageman R. H., 1972. Light saturation, photosynthesis rate, RuDP carboxylase activity and specific leaf weight in soybeans grown under different light intensities. Crop Sci., 12, 77.79.

Buchanan B. B., 1980. Role of light in the regulation of chloroplast enzymes. Annu. Rev. Plant Physiol., 31, 341-374.

Cavalié G., 1981. Respiration des végétaux verts : photorespiration. Soc. bot. Fr. : Journée d'étude sur la respiration des végétaux. 25 avril 1981.

Champigny M. L., 1980. Photorespiration et diversification des produits de la photosynthèse. Physiol. vég., 18 (4), 721-735.

Chen T. M., Brown R. H., Black C. C., 1971. Photosynthetic ${ }^{14} \mathrm{CO}_{2}$ fixation products and activities of enzymes related to photosynthesis in Bermudagrass and other plants. Plant Physiol., 47 (2), 199-203

Coïc Y., Lesaint C., 1973. La nutrition minérale en horticulture avancée. Rev. hortic., 23 (16), 29-34.

Coudret A., Ferron F., 1982. Régulation et dé-régulation du métabolisme photosynthétique en réponse aux variations du milieu chez des végétaux de type $C_{3}$. Agronomie, 2 (5), 429-436.

Coudret A., Louguet P., 1980. Etude comparée de l'action du $\mathrm{NaCl}$ sur les mouvements stomatiques de Plantago maritima L. et de Plantago lanceolata L. Physiol. vég., 18 (1), 55-68

Coudret A., Ferron F., Gaudillère J. P., Costes C., 1980. Action comparée des antitranspirants sur le mouvement des stomates : les échanges de $\mathrm{CO}_{2}$ et la production de matière sèche chez Plantago lanceolata L. et Plantago maritima L. Physiol. vég., 18 (4), 631-643.

Coudre1 A.. Ferron F., Gaudillere A. P.. 1981. Photosmblantes formation in wheat under different partial oxygen pressures and temperatures. Photosynthetica, 15 (1), 21-27.

Ferron F., Coudret A., Gaudillère J. P., 1978. Echanges et modes de fixation du gaz carbonique chez Plantago maritima L. et Plantago lanceolata $\mathrm{L}$. sous l'action de la salinité du milieu de culture. Bull. Soc. bot. Fr., Actualités botaniques, 3-4, 189-198.

Ferron F., Coudret A., Zinsou C., Costes C., 1982. Action de l'énergie lumineuse incidente sur le métabolisme photosynthétique de la christophine (Sechium edule Swartz). Agronomie, 2 (7), 621627

Galmiche J. M., 1973. Carboxylation primaire chez les végétaux supérieurs. Etude cinétique à l'aide $\mathrm{du}^{14} \mathrm{CO}_{2}$. Thèse Doct. Etat Sci. Nat., Orsay, 177 p.

Galzin A. M., 1979. Activité glycolate-oxydase chez les blés : con trôle génétique de l'activité et de la régulation par les polyphénols. Thèse de Doct. Ing., INA-PG, $66 \mathrm{p}$.

Gaudillère J. P., 1979. Variabilité du rendement quantique de la photosynthèse des plantes supérieures. Recherches de facteurs limitants. Thèse Doct. Etat Sci. Nat., Orsay, 75 p.

Hartree E. F., 1972. Determination of protein, a modification of the Lowry method that gives a linear photometric response. Anal. Biochem., 48 (2), 422-427.

Heber U., Krause G. H., 1980. What is the physiological role of photorespiration? Trends Biochem. Sci., 32, 32-40.

Kuiper P. J. C., 1961. The effects of environmental factors on the transpiration of leaves, with special reference to stomatal light response. Meded. Landbouwhogesch. Wageningen, 61, 1-49.

Louguet P., 1965. Sur une méthode d'étude du mouvement des stomates utilisant la diffusion de l'hydrogène à travers les feuilles. Actes Coll. Montpellier, UNESCO, 307-316.

Lowry O. H., Rosebrough N. J., Farr A. L., Randall R. J., 1951 Protein measurement with the Folin phenol reagent. J. Biol. Chem. $193,262-275$.

Mac Kinney G., 1941. Absorption of light by chlorophyll solutions. J. Biol. Chem., 140, 315-322.

Osmond C. B., 1981. Photorespiration and photoinhibition. Some implications for the energetics of photosynthesis. Biochim. Biophys. Acta, 639, 77-98.

Passera C., Albuzio A., 1978. Effect of salinity on photosynthesis and photorespiration of two wheat species (Triticum durum and Triticum aestivum). Can. J. Bot., 56, 312-316.

Powles S. B., Cornic G., Louason G., 1984. Photoinhibition of in vivo photosynthesis induced by strong light in the absence of $\mathrm{CO}_{2}$ : an appraisal of the hypothesis that photorespiration protects agains photoinhibition. Physiol. vég., 22 (4), 437-446.

Schürmann P., 1969. Separation of phosphate esters and algal extracts by thinlayer electrophoresis and chromatography. $J$. Chromatog., 39, 507-509.

Tolbert N. E., 1971. Leaf peroxisomes and photorespiration, 458-471. In Hatch M. D., Osmond C. B. \& Slatyer R. O. : "Photosynthesis and Photorespiration ». Wiley-Interscience, $565 \mathrm{p}$.

Wild A., 1979. Physiology and photosynthesis in higher plants. The adaptation to light intensity and light quality. Ber. Deutsch. Bot Ges., 92, 341-364. 